\title{
Hidden charm pentaquarks and tetraquark states
}

\section{Er-Liang Cui, Jia-Bing Xiang, Hua-Xing Chen*}

School of Physics and Beijing Key Laboratory of Advanced Nuclear Materials and Physics, Beihang University, Beijing 100191, China

E-mail: erliangcui.phy@buaa.edu.cn, xjbbuaa@buaa .edu.cn, hxchen@buaa.edu.cn

\section{Wei Chen}

School of Physics, Sun Yat-Sen University, Guangzhou 510275, China

E-mail: chenwei29@mail.sysu.edu.cn

\section{Xiang Liu}

School of Physical Science and Technology, Lanzhou University, Lanzhou 730000, China

E-mail: xiangliualzu.edu.cn

\section{Shi-Lin Zhu}

School of Physics and State Key Laboratory of Nuclear Physics and Technology and Collaborative Innovation Center of Quantum Matter,

Peking University, Beijing 100871, China

E-mail: zhusl@pku.edu.cn

We use the method of QCD sum rules to systematically study the mass spectrum of hidden-charm pentaquarks of spin $J=\frac{1}{2} / \frac{3}{2} / \frac{5}{2}$ and with the quark content $u u d c \bar{c}$. Our results suggest that the $P_{c}(4380)$ and $P_{c}(4450)$ can be identified as hidden-charm pentaquark states having $J^{P}=3 / 2^{-}$ and $5 / 2^{+}$, respectively.

XVII International Conference on Hadron Spectroscopy and Structure - Hadron2017

25-29 September, 2017

University of Salamanca, Salamanca, Spain

${ }^{*}$ Speaker. 


\section{Introduction}

Since the discovery of the $X(3872)$ in 2003 [1], dozens of charmonium-like $X Y Z$ states have been reported in recent years [2]. They are good candidates of tetraquark states, consisting of two quarks and two antiquarks. Two years ago in 2015, the LHCb Collaboration observed two hiddencharm pentaquark resonances, $P_{c}(4380)$ and $P_{c}(4450)$, in the $J / \psi p$ invariant mass spectrum [3]. They are good candidates of pentaquark states, consisting of four quarks and one antiquark. All the above states are exotic candidates, which can not be easily explained in the traditional quark model. They are of particular importance to understand the low energy behaviours of Quantum Chromodynamics (QCD).

There have been extensive theoretical studies on the existence of hidden-charm pentaquark states, and various theoretical methods and models have been applied to explain the nature of the $P_{c}(4380)$ and $P_{c}(4450)$, such as meson-baryon molecular states [4, 5, 6, 7], compact pentaquark states $[8,9]$, and kinematical effects related to the triangle singularity [10, 11], etc. We refer interested readers to reviews $[12,13,14,15,16]$ for detailed discussions. We have also used the method of QCD sum rules $[17,18,19]$ to study the mass spectrum of hidden-charm pentaquarks having spin $J=\frac{1}{2} / \frac{3}{2} / \frac{5}{2}$ and isospin $I=\frac{1}{2}$. In this paper we briefly introduce our method and results.

\section{Local Pentaquark Currents}

We have systematically constructed all the local hidden-charm pentaquark interpolating currents having spin $J=\frac{1}{2} / \frac{3}{2} / \frac{5}{2}$ and the quark contents $u u d c \bar{c}$ in Refs. [20, 21, 22]. We briefly summarize the results here. There are three possible color configurations, $\left[\bar{c}_{d} c_{d}\right]\left[\varepsilon^{a b c} u_{a} u_{b} d_{c}\right]$, $\left[\bar{c}_{d} u_{d}\right]\left[\varepsilon^{a b c} c_{a} u_{b} d_{c}\right]$, and $\left[\bar{c}_{d} d_{d}\right]\left[\varepsilon^{a b c} c_{a} u_{b} u_{c}\right]$, where $a \cdots d$ are color indices, $u, d$, and $c$ represent the up, down and charm quarks, respectively. These three configurations, as if they are local, can be related by the Fierz transformation and the color rearrangement:

$$
\delta^{d e} \varepsilon^{a b c}=\delta^{d a} \varepsilon^{e b c}+\delta^{d b} \varepsilon^{a e c}+\delta^{d c} \varepsilon^{a b e} .
$$

The first configuration, $\left[\bar{c}_{d} c_{d}\right]\left[\varepsilon^{a b c} u_{a} u_{b} d_{c}\right]$, can be easily constructed based on our studies on the internal structure of three-quark baryons [23]. However, the sum rules obtained using these currents are not useful. Considering that the $P_{c}(4380)$ and $P_{c}(4450)$ have masses significantly larger than the threshold of $J / \psi$ and proton, but close to thresholds of $D / D^{*}$ and $\Lambda_{c} / \Sigma_{c} / \Sigma_{c}^{*}$, we also construct currents belonging to the other two configurations, $\left[\bar{c}_{d} u_{d}\right]\left[\varepsilon^{a b c} c_{a} u_{b} d_{c}\right]$ and $\left[\bar{c}_{d} d_{d}\right]\left[\varepsilon^{a b c} c_{a} u_{b} u_{c}\right]$. Especially, we find the following two mixing currents:

$$
\begin{aligned}
J_{\mu, 3 / 2-}= & \cos \theta_{1} \times \xi_{36 \mu}+\sin \theta_{1} \times \psi_{9 \mu} \\
= & \cos \theta_{1} \times\left[\varepsilon^{a b c}\left(u_{a}^{T} C \gamma_{v} \gamma_{5} d_{b}\right) \gamma_{v} \gamma_{5} c_{c}\right]\left[\bar{c}_{d} \gamma_{\mu} \gamma_{5} u_{d}\right] \\
& +\sin \theta_{1} \times\left[\varepsilon^{a b c}\left(u_{a}^{T} C \gamma_{v} u_{b}\right) \gamma_{v} \gamma_{5} c_{c}\right]\left[\bar{c}_{d} \gamma_{\mu} d_{d}\right] \\
J_{\mu v, 5 / 2+}= & \cos \theta_{2} \times \xi_{15 \mu v}+\sin \theta_{2} \times \psi_{4 \mu v} \\
= & \cos \theta_{2} \times\left[\varepsilon^{a b c}\left(u_{a}^{T} C \gamma_{\mu} \gamma_{5} d_{b}\right) c_{c}\right]\left[\bar{c}_{d} \gamma_{v} u_{d}\right] \\
& +\sin \theta_{2} \times\left[\varepsilon^{a b c}\left(u_{a}^{T} C \gamma_{\mu} u_{b}\right) c_{c}\right]\left[\bar{c}_{d} \gamma_{v} \gamma_{5} d_{d}\right]+\{\mu \leftrightarrow v\}
\end{aligned}
$$

Their sum rules can be used to well interpret the $P_{c}(4380)$ and $P_{c}(4450)$ as hidden-charm pentaquark states having $J^{P}=3 / 2^{-}$and $5 / 2^{+}$, respectively. 


\section{QCD sum rules analyses}

In this section we use $J_{\mu, 3 / 2-}$ and $J_{\mu v, 5 / 2+}$ to perform QCD sum rule analyses. Again, we briefly introduce our method here, but refer to Refs. [20, 21, 22] for details. Firstly, we assume $J_{\mu, 3 / 2-}$ and $J_{\mu v, 5 / 2+}$ couple to physical states through

$$
\begin{aligned}
\left\langle 0\left|J_{\mu, 3 / 2-}\right| X_{3 / 2-}\right\rangle & =f_{X_{3 / 2-}} u_{\mu}(p), \\
\left\langle 0\left|J_{\mu v, 5 / 2+}\right| X_{5 / 2+}\right\rangle & =f_{X_{5 / 2+}} u_{\mu v}(p) .
\end{aligned}
$$

Their relevant two-point correlation functions can be written as

$$
\begin{aligned}
\Pi_{\mu v, 3 / 2-}\left(q^{2}\right) & =i \int d^{4} x e^{i q \cdot x}\left\langle 0\left|T\left[J_{\mu, 3 / 2-}(x) \bar{J}_{v, 3 / 2-}(0)\right]\right| 0\right\rangle \\
& =\left(\frac{q_{\mu} q_{v}}{q^{2}}-g_{\mu v}\right)\left(q+M_{X_{3 / 2}-}\right) \Pi_{3 / 2-}\left(q^{2}\right)+\cdots, \\
\Pi_{\mu v \rho \sigma, 5 / 2+}\left(q^{2}\right) & =i \int d^{4} x e^{i q \cdot x}\left\langle 0\left|T\left[J_{\mu v, 5 / 2+}(x) \bar{J}_{\rho \sigma, 5 / 2+}(0)\right]\right| 0\right\rangle \\
& =\left(g_{\mu \rho} g_{v \sigma}+g_{\mu \sigma} g_{v \rho}\right)\left(q+M_{X_{5 / 2+}}\right) \Pi_{5 / 2+}\left(q^{2}\right)+\cdots,
\end{aligned}
$$

where $\cdots$ contains non-relevant spin components.

At the hadron level, we can use the dispersion relation to rewrite them as

$$
\Pi\left(q^{2}\right)=\frac{1}{\pi} \int_{s_{<}}^{\infty} \frac{\operatorname{Im} \Pi(s)}{s-q^{2}-i \varepsilon} d s,
$$

where $s_{<}$is the physical threshold. We can evaluate its imaginary part by inserting intermediate hadron states $\sum_{n}|n\rangle\langle n|$, but adopting the usual parametrization of one-pole dominance for the ground state $X$ together with a continuum contribution:

$$
\begin{aligned}
\rho(s) \equiv \frac{1}{\pi} \operatorname{Im} \Pi(s) & =\sum_{n} \delta\left(s-M_{n}^{2}\right)\langle 0|J| n\rangle\langle n|\bar{J}| 0\rangle \\
& =f_{X}^{2} \delta\left(s-m_{X}^{2}\right)+\text { continuum. }
\end{aligned}
$$

At the quark and gluon level, we can calculate the two-point correlation functions (3.3-3.4) using the method of operator product expansion (OPE). After performing the Borel transform at both the hadron and quark-gluon levels, we can express the two-point correlation function as

$$
\Pi^{(a l l)}\left(M_{B}^{2}\right) \equiv \mathscr{B}_{M_{B}^{2}} \Pi\left(p^{2}\right)=\int_{s_{<}}^{\infty} e^{-s / M_{B}^{2}} \rho(s) d s .
$$

After assuming that the continuum contribution can be well approximated by the OPE spectral density above a threshold value $s_{0}$, we obtain the following sum rule relation

$$
M_{X}^{2}\left(s_{0}, M_{B}\right)=\frac{\int_{s_{<}}^{s_{0}} e^{-s / M_{B}^{2}} \rho(s) s d s}{\int_{s_{<}}^{s_{0}} e^{-s / M_{B}^{2}} \rho(s) d s} .
$$

We use the mixing current $J_{\mu, 3 / 2-}$ defined in Eq. (2.2) to perform sum rule analyses. The detailed sum rule expression has been give in Ref. [22]. We find that the terms proportional to $\mathbf{1} \times g_{\mu \nu}$ are almost the same as those proportional to $q \times g_{\mu \nu}$, suggesting that the state coupled by $J_{\mu, 3 / 2-}$ has the spin-parity $J^{P}=3 / 2^{-}$[24]. Similarly, we use $J_{\mu v, 5 / 2+}$ defined in Eq. (2.3) to perform sum rule analyses. These two sum rules will be used to perform numerical analyses in the next section. 


\section{Numerical Analyses}

In this section we use $J_{\mu, 3 / 2-}$ and $J_{\mu v, 5 / 2+}$ to perform numerical analyses. Various condensates inside their sum rules take the following values $[2,25,26,27,28,29,30,31]$ :

$$
\begin{aligned}
& \langle\bar{q} q\rangle=-(0.24 \pm 0.01)^{3} \mathrm{GeV}^{3}, \\
& \left\langle g_{s}^{2} G G\right\rangle=(0.48 \pm 0.14) \mathrm{GeV}^{4}, \\
& \left\langle g_{s} \bar{q} \sigma G q\right\rangle=M_{0}^{2} \times\langle\bar{q} q\rangle, \\
& M_{0}^{2}=-0.8 \mathrm{GeV}^{2} . \\
& m_{c}=1.275 \pm 0.025 \mathrm{GeV} .
\end{aligned}
$$

There are altogether three free parameters: the mixing angles $\theta_{1 / 2}$, the Borel mass $M_{B}$, and the threshold value $s_{0}$. We use the following three criteria to constrain them:

1. To insure the convergence of the OPE series, we require the dimension eight to be less than $10 \%$, which can be used to determine the lower limit of the Borel mass:

$$
\mathrm{CVG} \equiv\left|\frac{\prod_{\langle\bar{q} q\rangle\left\langle g_{s} \bar{q} \sigma G q\right\rangle}\left(\infty, M_{B}\right)}{\Pi\left(\infty, M_{B}\right)}\right| \leq 10 \%
$$

2. To insure the one-pole parametrization to be valid, we require the pole contribution (PC) to be larger than or around $30 \%$, which can be used to determine the upper limit of the Borel mass:

$$
\mathrm{PC}\left(s_{0}, M_{B}\right) \equiv \frac{\Pi\left(s_{0}, M_{B}\right)}{\Pi\left(\infty, M_{B}\right)} \gtrsim 30 \%
$$

3. To obtain reliable mass predictions, we require that the $s_{0}, M_{B}$ and $\theta_{1 / 2}$ dependence of the mass prediction be the weakest.

We use the sum rules for the current $J_{\mu, 3 / 2-}$ as an example. Firstly, we fix $\theta_{1}=-42^{\circ}$ and $s_{0}=23 \mathrm{GeV}^{2}$, and find that: a) the OPE convergence improves with the increase of $M_{B}$, and the first criterion requires that $M_{B}^{2} \geq 2.89 \mathrm{GeV}^{2}$, and b) the PC decreases with the increase of $M_{B}$, and $\mathrm{PC}=32 \%$ when $M_{B}^{2}=2.89 \mathrm{GeV}^{2}$. Accordingly, we fix the Borel mass to be $M_{B}^{2}=2.89 \mathrm{GeV}^{2}$ and choose $2.59 \mathrm{GeV}^{2}<M_{B}^{2}<3.19 \mathrm{GeV}^{2}$ as our working region. We show variations of $M_{X}$ with respect to $M_{B}$ in the left panel of Fig. 1, and find that the mass curves are quite stable inside this region.

To determine $s_{0}$, we show variations of $M_{X}$ with respect to $s_{0}$ in the middle panel of Fig. 1 when fixing $\theta_{1}=-42^{\circ}$. The $s_{0}$ dependence of the mass prediction is the weakest when $s_{0}$ is around 17 $\mathrm{GeV}^{2}$, but the pole contribution at this point is too small (just $8 \%$ ). We find that $\mathrm{PC}=32 \%$ at $s_{0}=23 \mathrm{GeV}^{2}$, where the $M_{B}$ dependence is the weakest. Accordingly, we fix the threshold value to be $s_{0}=23 \mathrm{GeV}^{2}$ and choose $21 \mathrm{GeV}^{2} \leq s_{0} \leq 25 \mathrm{GeV}^{2}$ as our working region.

Finally, we change $\theta_{1}$ and redo the above processes. We show variations of $M_{X}$ with respect to $\theta_{1}$ in the right panel of Fig. 1 when fixing $s_{0}=23 \mathrm{GeV}^{2}$ and choosing $M_{B}$ to satisfy the first criterion. We find that the $\theta_{1}$ dependence of the mass prediction is weak when $\theta_{1} \leq-40^{\circ}$. Accordingly, we fix the mixing angle $\theta_{1}$ to be $-42^{\circ}$ and choose $\theta_{1}=-42 \pm 5^{\circ}$ as our working region. 


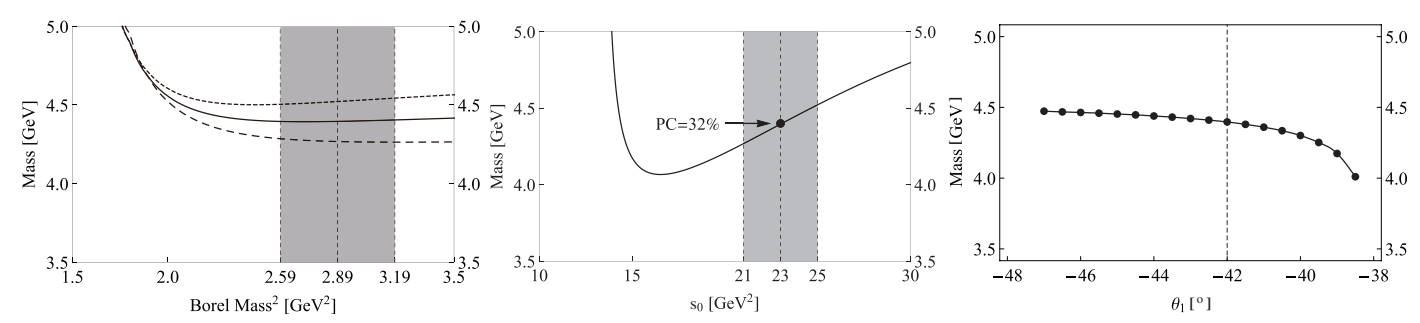

Figure 1: Variations of $M_{3 / 2^{-}}$with respect to the Borel mass $M_{B}$ (left), the threshold value $s_{0}$ (middle) and the mixing angle $\theta_{1}$ (right), calculated using the current $J_{\mu, 3 / 2-}$ of $J^{P}=3 / 2^{-}$.

Altogether, the working regions are found to be $21 \mathrm{GeV}^{2} \leq s_{0} \leq 25 \mathrm{GeV}^{2}, 2.59 \mathrm{GeV}^{2}<M_{B}^{2}<$ $3.19 \mathrm{GeV}^{2}$, and $-47^{\circ}<\theta_{1}<-37^{\circ}$, where we obtain the following numerical results:

$$
\begin{aligned}
M_{3 / 2^{-}} & =4.40_{-0.22}^{+0.17} \mathrm{GeV}, \\
f_{3 / 2^{-}} & =\left(6.5_{-2.9}^{+3.2}\right) \times 10^{-4} \mathrm{GeV}^{6} .
\end{aligned}
$$

Here the mass uncertainty is due to the mixing angle $\theta_{1}$, the Borel mass $M_{B}$, the threshold value $s_{0}$, the charm quark mass $m_{c}$, and various condensates. This mass value is consistent with the experimental mass of the $P_{c}(4380)$, and supports it to be a hidden-charm pentaquark having $J^{P}=$ $3 / 2^{-}$.

Similarly, we use the current $J_{\mu v, 5 / 2+}$ of $J^{P}=5 / 2^{+}$to perform numerical analyses, and obtain the following numerical results:

$$
\begin{aligned}
M_{5 / 2^{+}} & =4.50_{-0.24}^{+0.26} \mathrm{GeV}, \\
f_{5 / 2^{+}} & =\left(5.5_{-2.4}^{+3.4}\right) \times 10^{-4} \mathrm{GeV}^{6},
\end{aligned}
$$

This mass value is consistent with the experimental mass of the $P_{c}(4450)$, and supports it to be a hidden-charm pentaquark having $J^{P}=5 / 2^{+}$.

\section{Summary and discussions}

In this paper we use the method of QCD sum rules to study the hidden-charm pentaquark states $P_{c}(4380)$ and $P_{c}(4450)$. After systematically constructing all the local hidden-charm pentaquark interpolating currents of spin $J=\frac{1}{2} / \frac{3}{2} / \frac{5}{2}$ and with the quark contents $u u d c \bar{c}$, we find two mixing currents, $J_{\mu, 3 / 2-}$ of $J^{P}=3 / 2^{-}$and $J_{\mu v, 5 / 2+}$ of $J^{P}=5 / 2^{+}$. We use them to perform QCD sum rule analyses and obtain

$$
\begin{aligned}
& M_{3 / 2^{-}}=4.40_{-0.23}^{+0.16} \mathrm{GeV} \\
& M_{5 / 2^{+}}=4.50_{-0.23}^{+0.26} \mathrm{GeV}
\end{aligned}
$$

These values are consistent with the experimental masses of the $P_{c}(4380)$ and $P_{c}(4450)$, suggesting that the $P_{c}(4380)$ and $P_{c}(4450)$ can be well interpreted as hidden-charm pentaquark states having $J^{P}=3 / 2^{-}$and $5 / 2^{+}$, respectively. 
We have also investigated their bottom partners, the hidden-bottom pentaquark states ( $b \bar{b} u u d)$ of $J^{P}=3 / 2^{-}$and $J^{P}=5 / 2^{+}$. We use the method of QCD sum rules to evaluate their masses to be

$$
\begin{aligned}
& M_{P_{b}\left(3 / 2^{-}\right)}=10.83_{-0.29}^{+0.26} \mathrm{GeV}, \\
& M_{P_{b}\left(5 / 2^{+}\right)}=10.85_{-0.27}^{+0.24} \mathrm{GeV},
\end{aligned}
$$

and propose to search for them in future $\mathrm{LHCb}$ and BelleII experiments.

\section{ACKNOWLEDGMENTS}

This project is supported by the National Natural Science Foundation of China under Grants No. 11722540 , No. 11175073 , No. 11205011, No. 11222547, No. 11375024 , No. 11475015 , No. 11575008, No. 11261130311, and No. 11621131001, the 973 program, the Ministry of Education of China (SRFDP under Grant No. 20120211110002 and the Fundamental Research Funds for the Central Universities), the Fok Ying-Tong Education Foundation (Grant No. 131006), and the National Program for Support of Top-notch Young Professionals.

\section{References}

[1] S. K. Choi et al. [Belle Collaboration], Observation of a narrow charmonium-like state in exclusive $B^{ \pm} \rightarrow K^{ \pm} \pi^{+} \pi^{-} J / \psi$ decays, Phys. Rev. Lett. 91, 262001 (2003) [hep-ex/0309032].

[2] C. Patrignani et al. [Particle Data Group], Review of Particle Physics, Chin. Phys. C 40, 100001 (2016).

[3] R. Aaij et al. [LHCb Collaboration], Observation of J/ $\psi p$ Resonances Consistent with Pentaquark States in $\Lambda_{b}^{0} \rightarrow J / \psi K^{-} p$ Decays, Phys. Rev. Lett. 115, 072001 (2015) [arXiv: 1507.03414 [hep-ex]].

[4] J. J. Wu, R. Molina, E. Oset and B. S. Zou, Prediction of narrow $N^{*}$ and $\Lambda^{*}$ resonances with hidden charm above 4 GeV, Phys. Rev. Lett. 105, 232001 (2010) [arXiv: 1007.0573 [nucl-th]].

[5] M. Karliner and J. L. Rosner, New Exotic Meson and Baryon Resonances from Doubly-Heavy Hadronic Molecules, Phys. Rev. Lett. 115, 122001 (2015) [arXiv: 1506.06386 [hep-ph] ].

[6] R. Chen, X. Liu, X. Q. Li and S. L. Zhu, Identifying exotic hidden-charm pentaquarks, Phys. Rev. Lett. 115, 132002 (2015) [arXiv: 1507.03704 [hep-ph] ].

[7] U. G. Meissner and J. A. Oller, Testing the $\chi_{c 1} p$ composite nature of the $P_{c}(4450)$, Phys. Lett. $B$ 751, 59 (2015) [arXiv:1507.07478 [hep-ph]].

[8] L. Maiani, A. D. Polosa and V. Riquer, The New Pentaquarks in the Diquark Model, Phys. Lett. B 749, 289 (2015) [arXiv:1507.04980 [hep-ph]].

[9] R. F. Lebed, The Pentaquark Candidates in the Dynamical Diquark Picture, Phys. Lett. B 749, 454 (2015) [arXiv: 1507.05867 [hep-ph]].

[10] F. K. Guo, Ulf-G. Meissner, W. Wang and Z. Yang, How to reveal the exotic nature of the $P_{c}(4450)$, Phys. Rev. D 92, 071502 (2015) [arXiv:1507.04950 [hep-ph] ].

[11] X. H. Liu, Q. Wang and Q. Zhao, Understanding the newly observed heavy pentaquark candidates, Phys. Lett. B 757, 231 (2016) [arXiv: 1507.05359 [hep-ph] ]. 
[12] H. X. Chen, W. Chen, X. Liu and S. L. Zhu, The hidden-charm pentaquark and tetraquark states, Phys. Rept. 639, 1 (2016) [arXiv:1601.02092 [hep-ph]].

[13] H. X. Chen, W. Chen, X. Liu, Y. R. Liu and S. L. Zhu, A review of the open charm and open bottom systems, Rept. Prog. Phys. 80, 076201 (2017) [arXiv:1609.08928 [hep-ph]].

[14] A. Esposito, A. Pilloni and A. D. Polosa, Multiquark Resonances, Phys. Rept. 668, 1 (2016) [arXiv:1611.07920 [hep-ph]].

[15] F. K. Guo, C. Hanhart, U. G. Meissner, Q. Wang, Q. Zhao and B. S. Zou, Hadronic molecules, arXiv:1705.00141 [hep-ph].

[16] S. L. Olsen, T. Skwarnicki and D. Zieminska, Non-Standard Heavy Mesons and Baryons, an Experimental Review, arXiv:1708.04012 [hep-ph].

[17] M. A. Shifman, A. I. Vainshtein and V. I. Zakharov, QCD And Resonance Physics. Sum Rules, Nucl. Phys. B 147, 385 (1979).

[18] L. J. Reinders, H. Rubinstein and S. Yazaki, Hadron Properties From QCD Sum Rules, Phys. Rept. 127, 1 (1985).

[19] M. Nielsen, F. S. Navarra and S. H. Lee, New Charmonium States in QCD Sum Rules: A Concise Review, Phys. Rept. 497, 41 (2010) [arXiv:0911.1958 [hep-ph] ].

[20] H. X. Chen, W. Chen, X. Liu, T. G. Steele and S. L. Zhu, Towards exotic hidden-charm pentaquarks in QCD, Phys. Rev. Lett. 115, 172001 (2015) [arXiv:1507.03717 [hep-ph] ].

[21] H. X. Chen, E. L. Cui, W. Chen, X. Liu, T. G. Steele and S. L. Zhu, QCD sum rule study of hidden-charm pentaquarks, Eur. Phys. J. C 76, 572 (2016) [arXiv:1602.02433 [hep-ph] ].

[22] J. B. Xiang, H. X. Chen, W. Chen, X. B. Li, X. Q. Yao and S. L. Zhu, Revisiting hidden-charm pentaquarks from QCD sum rules, arXiv: 1711.01545 [hep-ph] .

[23] H. X. Chen, V. Dmitrasinovic, A. Hosaka, K. Nagata and S. L. Zhu, Chiral Properties of Baryon Fields with Flavor SU(3) Symmetry, Phys. Rev. D 78, 054021 (2008) [arXiv: 0806.1997 [hep-ph]].

[24] K. Ohtani, P. Gubler and M. Oka, Parity projection of QCD sum rules for the nucleon, Phys. Rev. D 87, 034027 (2013) [arXiv:1209.1463 [hep-ph]].

[25] M. Eidemuller and M. Jamin, Charm quark mass from QCD sum rules for the charmonium system, Phys. Lett. B 498, 203 (2001) [hep-ph/ 0010334 ].

[26] S. Narison, QCD as a theory of hadrons (from partons to confinement), Camb. Monogr. Part. Phys. Nucl. Phys. Cosmol. 17, 1 (2002).

[27] V. Gimenez, V. Lubicz, F. Mescia, V. Porretti and J. Reyes, Operator product expansion and quark condensate from lattice QCD in coordinate space, Eur. Phys. J. C 41, 535 (2005) [hep-lat/0503001].

[28] M. Jamin, Flavour-symmetry breaking of the quark condensate and chiral corrections to the Gell-Mann-Oakes-Renner relation, Phys. Lett. B 538, 71 (2002) [hep-ph/0201174].

[29] B. L. Ioffe and K. N. Zyablyuk, Gluon condensate in charmonium sum rules with 3-loop corrections, Eur. Phys. J. C 27, 229 (2003) [hep-ph/0207183].

[30] A. A. Ovchinnikov and A. A. Pivovarov, QCD Sum Rule Calculation Of The Quark Gluon Condensate, Sov. J. Nucl. Phys. 48, 721 (1988) [Yad. Fiz. 48, 1135 (1988)].

[31] P. Colangelo and A. Khodjamirian, At the Frontier of Particle Physics/Handbook of QCD, World Scientific, Singapore, 2001. 